Cahiers $d u$ MONDE RUSSE

\section{Cahiers du monde russe}

Russie - Empire russe - Union soviétique et États indépendants

$46 / 4 \mid 2005$

L'invention d'une politique humanitaire

\title{
Le placement des réfugiés russes dans l'agriculture
}

L'état français et le zemgor dans l'action internationale

Catherine Gousseff

\section{Q OpenEdition}

1 Journals

Édition électronique

URL : https://journals.openedition.org/monderusse/9430

DOI : $10.4000 /$ monderusse. 9430

ISSN : $1777-5388$

Éditeur

Éditions de l'EHESS

\section{Édition imprimée}

Date de publication : 1 décembre 2005

Pagination : 757-776

ISBN : 2-7132-2057-2

ISSN : $1252-6576$

\section{Référence électronique}

Catherine Gousseff, «Le placement des réfugiés russes dans l'agriculture », Cahiers du monde russe [En ligne], 46/4 | 2005, mis en ligne le 01 janvier 2005, consulté le 02 septembre 2022. URL : http:// journals.openedition.org/monderusse/9430; DOI : https://doi.org/10.4000/monderusse.9430 


\section{LE PLACEMENT DES RÉFUGIÉS RUSSES DANS L'AGRICULTURE}

\section{L'État français et le Zemgor dans l'action internationale}

Dans l'histoire des collaborations internationales mises en place au lendemain de la guerre civile pour résoudre les problèmes posés par l'afflux de réfugiés, les actions dites de «placement» professionnel, qui consistaient à transférer des Russes précairement accueillis dans les États d'Europe centrale et orientale vers les centres industriels ou agricoles français, ont concentré de très importantes énergies. Elles ont également suscité des synergies jusqu'alors inédites entre États, organisations internationales et organisations privées russes, configurant ainsi l'une des grandes caractéristiques de l'action humanitaire moderne. L'étude de cas retenue, le placement des réfugiés russes dans les régions agricoles du Sud-Ouest français constitue un terrain d'analyse multiple. Il permet de retracer l'investissement spécifique du Zemgor dans l'assistance par le travail et le rôle qu'il fut amené à jouer dans l'immigration dirigée des Russes vers les terroirs français. Il illustre l'idéal contenu dans la figure de la colonisation agricole qui a fait du projet de recrutement des réfugiés un enjeu de reclassement social et d'insertion ambitieux, nécessitant dans les modalités techniques de sa réalisation ajustements et inventivité. Il permet, enfin, de suivre les formes de collaboration, mais aussi de dysfonctionnement, dans la conduite d'une entreprise mêlant des protagonistes aux statuts très différents. Quelle fut la place dévolue au Zemgor par les représentants institutionnels du projet, le ministère de l'Agriculture et le Bureau international du travail ? La répartition des fonctions, la définition des modes de relations entre acteurs privés et acteurs publics ont été à la source de plusieurs différends. Ceux-ci ont, certes, porté sur le rôle des organisations russes, mais aussi sur celui des entreprises commer-

Dans cet article, la graphie retenue pour les noms russes est celle des documents d'archives consultés. 
ciales de recrutement, déclinant sous des problématiques très distinctes l'intervention des sphères privées dans le projet. En outre, ces contentieux ont opposé non pas tant les Russes aux partenaires institutionnels, que les partenaires institutionnels entre eux. L'histoire des réfugiés et de la colonisation agricole rend compte du défi posé par la promotion de la coopération humanitaire dans la quête de normes et de définition de l'intérêt public.

\section{L'improvisation des premières années et le rôle pionnier du Zemgor}

En 1921, lors de sa constitution en association, le Zemgor s'est imposé, avec la Croix-Rouge russe, comme la plus grande des organisations d'assistance de l'émigration russe. Ses atouts étaient multiples. Dirigé par les libéraux de la révolution de Février 1917, il rassemblait l'aile démocratique d'un mouvement antibolchevik politiquement très hétérogène et représentait, pour ainsi dire, le bras opérationnel du Conseil des ambassadeurs grâce auquel il fut pourvu, dans les premières années du moins, d'importantes sources de financement. En tant qu'organisation civile et démocratique, le Zemgor bénéficia du soutien de nombreux interlocuteurs extérieurs impliqués dans l'aide aux Russes exilés, en premier lieu de celui des autorités françaises qui étaient alors les plus engagées dans la prise en charge des réfugiés évacués vers la Turquie ${ }^{1}$ et, en second lieu, de celui du Haut commissariat pour les réfugiés russes (HCR), instance créée à l'été 1921 au sein de la Société des Nations, sous la présidence de Fridjtof Nansen, avec pour mission de coordonner l'aide intergouvernementale et associative en faveur des déshérités de la révolution, alors évalués à au moins deux millions de personnes ${ }^{2}$.

Rassemblant les anciens animateurs des administrations locales russes, très nombreux en exil, le Comité directeur parisien du Zemgor pouvait s'appuyer sur un réseau important d'hommes compétents, aguerris aux pratiques de gestion et qui avaient hérité de leur engagement para-étatique au cours de la Grande Guerre, une expérience spécifique dans l'aide humanitaire. Il résulta de ce réseau la constitution en 1921 de 17 filiales de l'organisation représentée dans pratiquement tous les pays d'accueil de l'émigration ${ }^{3}$. Dès cette date, le Zemgor pouvait donc prétendre à une action véritablement étendue à tous les pays d'accueil.

$\mathrm{Au}$ moment du changement de statut du Zemgor, la situation des réfugiés massés dans les pays limitrophes de la Russie était partout précaire et instable, mais

1. L'entremise des autorités françaises dans la constitution en association du Zemgor est indirectement attestée (voir l'article de I. Savický dans ce même numéro), mais elle reste cependant à préciser.

2. Des travaux ultérieurs effectués en particulier par l'équipe de spécialistes réunie autour de J.H. Simpson conduiront à réduire les premières estimations pour une nouvelle évaluation oscillant entre 600000 à 750000 Russes exilés au début des années 1920. John Hope Simpson, The Refugee Problem: Report of a Survey, Londres : Oxford University Press, 1939.

3. Le Zemgor était représenté en France, Grande-Bretagne, Allemagne, Finlande, Estonie, Lettonie, Lituanie, Tchécoslovaquie, Pologne, Suisse, Yougoslavie, Bulgarie, Turquie, Égypte, Grèce et sur l'île de Chypre. 
c'est surtout dans le Bosphore que les cris d'alarme se multipliaient. L'essor du mouvement kémaliste, le conflit gréco-turc qui allait bientôt aboutir à un échange inédit de populations faisaient de la région une véritable poudrière. L'évacuation des réfugiés s'imposait, mais l'incertitude sur le devenir de la Russie soviétique contrariait les perspectives. Les lieutenants de l'Armée blanche escomptaient toujours une chute prochaine du régime et, pour des raisons stratégiques, cherchaient à demeurer à proximité du pays, influençant le comportement de nombreux civils réticents à quitter les régions frontalières de la Russie. De son côté, l'étatmajor français basé à Constantinople encourageait les rapatriements des troupes évacuées, anticipant en cela la politique qui sera suivie par le Haut commissariat pour les réfugiés russes dans les années suivantes à l'égard des vétérans de l'Armée blanche et de leurs familles. Dès sa prise de fonction, le haut commissaire Fridjtof Nansen avait envisagé comme alternative au retour des réfugiés en Russie leur envoi massif vers les pays d'Amérique latine dans le cadre de vastes projets de colonisation agricole. Autant dire que, dans cette première période de l'exil, l'installation durable des réfugiés en Europe ne constituait guère le souci majeur des différents acteurs de l'assistance. Seul le Zemgor préconisa ouvertement et très précocement cette orientation : « le Comité considère comme l'un des problèmes les plus importants qui soient à résoudre la répartition des réfugiés en des pays et des régions où ils pourraient trouver un travail permanent et rétribué $»^{4}$, proclamaitil dès le printemps 1921. Sans se prononcer sur les pays d'installation visés, le Comité annonçait à la suite la création de bureaux de travail (bjuro truda) à Paris et Marseille (avec des filiales à Lille et Grenoble), profilant ainsi l'action de placement qu'allaient mener les animateurs du Zemgor tout au long de la décennie. Les bureaux de travail en question furent fondés et gérés par l'association des membres des zemstvos et des municipalités, branche locale « française », du Comité directeur, qui se constitua en intermédiaire entre l'offre des entreprises et les demandes d'emplois des réfugiés ${ }^{5}$. Lors de son premier bilan d'activité en mai 1922, le Zemgor indiquait avoir contribué à l'embauche de plus de 4000 personnes $^{6}$. Ce résultat était non négligeable dans une période où les migrations vers la France demeuraient encore très minoritaires.

Jusqu'en 1922, la circulation des réfugiés depuis l'Europe centrale et balkanique était subordonnée à la détention de papiers d'identité et nombre d'émigrés en étaient dépourvus. Or l'instauration du système de visas mis en place par la France

4. Bulletin du Comité des zemstvos et des municipalités de secours aux citoyens russes à l'étranger, 1er mars 1921, n 1, p. 6 (Il existait déjà un bulletin du Comité édité en russe (Bjulleten' rossijskago zemsko-gorodskogo komiteta pomošči bežencam) dont le $1^{\text {er }}$ numéro était daté du 25 février 1921.

5. Nous n'avons malheureusement trouvé trace des archives de cette association, y compris aux Archives russes de Leeds où ont été déposés les fonds du Comité directeur du Zemgor. Il est donc impossible de retracer l'activité de ces bureaux de travail et, en particulier, de reconstituer les réseaux tissés avec les entreprises françaises qui auraient permis d'éclairer les résultats très positifs de cette action.

6. Rossiiskij zemsko-gorodskoj komitet, Otčet o dejatel'nosti (fev. 1921-aprel'1922), Paris, 1922, p. 90-91. 
et bientôt généralisé en Europe ne laissait aucune liberté de manœuvre aux «sans papiers ». C'est à cet obstacle que s'attaquèrent les premiers travaux du HCR, élaborant, de concert avec les gouvernements et les représentants de l'émigration russe, le premier certificat de réfugié russe qui vit officiellement le jour en juin 1922. Dans la plupart des cas, ce certificat était délivré par les agents locaux du Bureau international du travail (BIT), installé dans différentes capitales européennes. Dès la création du HCR, les compétences du BIT avaient été requises. Les très maigres subsides alloués par la SDN au fonctionnement du HCR avaient été précisément justifiés par la mise à disposition des services techniques déjà en place à Genève ${ }^{7}$. Dans un premier temps, le BIT fut surtout sollicité à titre d'expert avant d'intervenir directement dans l'action du HCR, en particulier à travers la délivrance des certificats de réfugié. Par le biais de cette nouvelle mission, les services locaux du BIT renforcèrent leurs contacts avec certaines des organisations ou comités russes qui s'étaient mis en place dans les différents pays de la diaspora et parmi lesquels figuraient à titre collectif ou individuel des représentants du Zemgor. La constitution, entre autres à Constantinople et Sofia, de réseaux entre les animateurs sociaux de l'émigration russe (obščestvennye dejateli) et les agents mandatés de Genève s'est ainsi concrétisée à travers la gestion des papiers des réfugiés. Ces noyaux locaux joueront par la suite un rôle important dans l'organisation du recrutement à distance des Russes en France.

La mise en place des certificats par F. Nansen marqua l'essor des grandes migrations russes à travers l'Europe, en premier lieu vers la France. Dès lors, l'activité du Zemgor relative au placement professionnel des réfugiés dans le pays ne cessa de s'intensifier. Fin 1923, le Zemgor revendiquait l'embauche par son entremise de plus de 30000 Russes $^{8}$. Ce bilan impressionnant dressé au terme de deux ans d'existence soulignait l'efficacité et la place occupée par l'organisation dans l'accueil des réfugiés. Mais c'est justement cette efficacité qui lui valut, fin 1923, un blâme de la part du service de la main-d'œuvre étrangère du ministère du Travail. Dans un courrier daté du 8 novembre, le Zemgor était invité à cesser ses activités de placement.

[...] D'après la loi, les contingents ouvriers ne peuvent être introduits en France qu'avec l'autorisation du ministère du Travail et c'est lui seul qui est compétent pour la répartition de ces ouvriers dans les diverses industries. Monsieur Lebelle (chef du service de la main-d'œuvre étrangère du ministère du Travail) m'a mis au courant des difficultés qu'il rencontrait avec les œuvres privées russes établies sur le territoire. Ces œuvres interviennent constamment auprès des employeurs pour obtenir le placement de leurs nationaux et entravent ainsi son

7. Voir à ce propos Dzovinar Kévonian, Réfugiés et diplomatie humanitaire : les acteurs européens et la scène proche-orientale pendant l'entre-deux-guerres, Paris : Publications de la Sorbonne, 2004.

8. Archives SDN, Fonds mixte Nansen (plus loin ASDN, FMN) C 1437, Rr 404/3/61/1, Lettre de M. Krovopouskoff (secrétaire de l'Association des membres des zemstvos et des municipalités de secours aux citoyens russes à l'étranger) à M. Hainglaise (représentant français mandaté auprès du HCR) du 12 novembre 1923. 
action. Il désirerait vivement que les œuvres privées fussent invitées à ne plus continuer ces pratiques ${ }^{9}$.

Dans sa réponse, le Zemgor souligna le malentendu existant, son activité ne consistait pas à organiser les migrations de réfugiés vers la France, mais à trouver du travail à ceux déjà arrivés légalement dans le pays. Néanmoins, l'interdiction de poursuivre son action lui fut fermement réitérée par Lebelle en personne ${ }^{10}$.

L'intervention impromptue des autorités françaises visait à conformer les modalités d'entrée des réfugiés à celles des autres étrangers. Depuis le début de la décennie, la pénurie de main-d'œuvre avait été à l'origine d'une immigration massive, mais en grande partie contrôlée. Les recrutements collectifs à distance, par le biais de contrats bilatéraux passés avec les pays d'émigration étaient devenus, pour ainsi dire, la norme ${ }^{11}$. L'arrivée massive des ouvriers polonais dans les mines du Nord en était l'un des exemples les plus spectaculaires ${ }^{12}$. Une partie significative des immigrés se présentait ainsi aux frontières munis d'un contrat d'embauche et c'est ce qui fut désormais exigé des émigrés russes.

Que motivait ce brusque revirement d'attitude ? D'après l'agent du BIT à Sofia, cette décision était liée au flux toujours plus important de réfugiés en provenance de l'Allemagne, pays déchiré par une crise monétaire et économique désastreuse ${ }^{13}$. Cependant, une autre préoccupation majeure s'ajoutait à la volonté de contenir une immigration qui risquait de devenir rapidement incontrôlable. À partir de 1923, l'état-major des armées blanches, qui avait tout d'abord encouragé le cantonnement de ses troupes dans les pays balkaniques, changea de tactique et entérina l'évolution en cours. La dégradation de la situation socio-économique des réfugiés dans ces pays était à l'origine d'importants départs spontanés vers l'Europe occidentale : il décida de les encadrer en favorisant, autant que possible, l'embauche de contingents entiers. L'entrée en scène des dirigeants militaires dans les opérations de placement était particulièrement mal perçue des autorités françaises et l'intervention du ministère du Travail auprès des organisations russes ne fut pas étrangère à cette nouvelle donne ${ }^{14}$. La crainte de voir s'établir sous couvert de groupes

9. Ibid., Lettre du 8 novembre 1923 de M. Hainglaise à M. Krovopouskoff.

10. Ibid., Réponse de M. Krovopouskoff à M. Hainglaise du 12 novembre 1923, Courrier de M. Lebelle à M. Krovopouskoff du 12 décembre 1923. Bien qu'il affirmât le contraire dans sa réponse, le Zemgor encourageait effectivement l'organisation des migrations vers la France. Il diffusait notamment dans les différents pays de la diaspora des formulaires relatifs à la formation des réfugiés et à leurs souhaits en matière d'emplois (voir par exemple, GARF [Gosudarstvennyj Arhiv Rossiskoj Federacii], f. 5771, op. 2, d. 23, enquête du Zemgor sur les profils professionnels des réfugiés russes de Yougoslavie).

11. André Pairault, L'immigration organisée et l'emploi de la main-d'œuvre étrangère en France, Paris : Presses universitaires de France, 1926.

12. J. Ponty, Polonais méconnus : Histoire des travailleurs immigrés en France dans l'entredeux-guerres, Paris : Publications de la Sorbonne, 1988.

13. ASDN, FMN, R 1749/45-29317, Courrier de Collins (représentant du BIT à Sofia) à Gorvin (BIT, Genève) du 19 novembre 1923.

14. Voir à ce propos Paul Robinson, The White Russian Army in Exile, 1920-1941, Oxford : Clarendon press, 2002, p. 87-90. 
d'ouvriers des bataillons prêts à repartir au front sera, nous le verrons, récurrente tout au long de la décennie.

L'obligation pour les Russes d'être en possession d'un contrat d'embauche afin d'obtenir un visa supposait que l'on procédât désormais à leur recrutement ${ }^{15}$. Les quelques contrats délivrés aux réfugiés par le Comité des houillères signalaient surtout l'intérêt porté à cette main-d'œuvre potentielle, mais aucune action d'envergure n'avait été engagée. La tâche n'était effectivement pas si facile. Contrairement aux travailleurs étrangers «contractuels », les Russes ne bénéficiaient d'aucune protection étatique à même de les représenter. La nécessité s'imposait d'inventer une autorité de substitution qui pût servir d'interlocuteur face aux employeurs en quête de main-d'œuvre. De ce point de vue, l'année 1924 fut décisive.

\section{L'organisation du placement des réfugiés et la répartition des rôles}

De 1921 à 1924, l'action engagée par le HCR à l'égard des Russes avait connu un succès très inégal. Les initiatives prises par F. Nansen pour résoudre le problème des masses de réfugiés démunis s'étaient soldées par des échecs, qu'il s'agisse de l'incitation au rapatriement (les amnisties proclamées par la RSFSR à l'encontre des « gardes blancs » avaient conforté cette option), ou de l'organisation de la colonisation agricole en Amérique latine ${ }^{16}$. En revanche, le HCR était parvenu à s'imposer sur la scène européenne à travers les concertations intergouvernementales qu'il avait animées, en particulier lors de la création du certificat de réfugié. Ce bilan guida de nouveaux choix. En 1924, F. Nansen abandonna l'idée du rapatriement des Russes et délégua les tâches de placement des réfugiés au BIT, ne remplissant plus désormais que ses fonctions de diplomate de l'humanitaire. C'est donc au BIT et à son président Albert Thomas qu'il revint d'organiser, en collaboration avec les pouvoirs publics français, les modalités d'embauche des Russes en France. Il fut convenu que les entreprises demandeuses de main-d'œuvre adresseraient leurs requêtes au BIT, qui transmettrait les propositions par l'intermédiaire de ses agents et prendrait en charge les procédures de recrutement (enregistrement des candidats, examen de leurs compétences, contrôle sanitaire, organisation des transports). Cette nouvelle charge excédait en partie les moyens des agents locaux

15. Celui-ci pouvait également être attribué si le requérant justifiait de moyens de subsistance suffisants, ce qui était évidemment très rarement le cas.

16. Un premier accord avait été passé avec les autorités brésiliennes pour organiser l'installation des Russes dans les plantations de café, mais le premier bateau en partance pour Sao Paulo, le Rion qui avait à son bord 3421 Russes venus des camps de Constantinople, Gallipoli et Lemnos, échoua en Corse pour des raisons techniques. Il y débarqua plus de la moitié des réfugiés. Beaucoup de ceux qui parvinrent au Brésil en revinrent par la suite (Rossijskij zemskogorodskoj komitet, Otčet o dejatel 'nosti (fev. 1921-aprel'1922), Paris, 1922, p. 98-99). Des pourparlers furent engagés avec les gouvernements sud-américains. Il en ressortit notamment que la question des documents d'identité et celle de l'expulsion problématique des réfugiés en cas de contentieux constituaient des obstacles majeurs. 
et contribua, de ce fait, à formaliser les liens existant dans différentes capitales entre les représentants du BIT et les organisations russes. A. Thomas lui-même encouragea ces collaborations locales. Il s'adressa à plusieurs filiales du Zemgor pour les inviter à prendre part aux opérations ${ }^{17}$. Dans certains cas, la création de sous-délégations du BIT donna lieu à la nomination d'émigrés russes à la tête de ces représentations (ce fut le cas de M. Yourieff à Belgrade, de M. Serafimoff à Sofia). Ceux-ci étaient invités à utiliser les réseaux associatifs russes pour faire connaître les modalités de recrutement.

Outre ces dispositions à l'échelle locale, l'organisation du placement collectif à distance posa sous un angle nouveau la question de la participation des organisations russes à l'œuvre d'ensemble. Dans un courrier adressé au secrétaire du Zemgor, A. Thomas revint longuement sur la fermeture obligée des bureaux de travail fin 1923, convenant que cette mesure avait été un «véritable gâchis ». Il faisait part des nouvelles dispositions arrêtées avec les pouvoirs publics français : la contribution des organisations russes à l'œuvre de placement était reconnue « tout à fait utile », mais, soulignait $\mathrm{A}$. Thomas, «c'est désormais à nous, et à nos agents que devront s'adresser les sociétés russes et, pour éviter concurrence et désordre, les employeurs français passeront par notre intermédiaire ${ }^{18}$. La position des Russes était ainsi clarifiée. Les acteurs institutionnels (services de main-d'œuvre par lesquels passaient d'ordinaire les employeurs, BIT) menaient le jeu, mais les sociétés russes pouvaient coopérer en adressant leurs requêtes et propositions au grand partenaire de l'œuvre humanitaire du HCR. Les dispositions adoptées avaient une évidente cohérence. Cependant, l'intervention d'un nouvel acteur compliqua la situation. La puissante Société générale d'immigration (SGI), créée en 1924 par un consortium de grosses entreprises françaises (dont notamment le Comité des houillères) allait très vite s'imposer comme la plus grande agence privée de recrutement de main-d'œuvre étrangère. Au cours des pourparlers entre le BIT et Lebelle, responsable de la main-d'œuvre étrangère au ministère du Travail, le rôle de la SGI dans l'embauche à distance des Russes était, de l'avis du ministère, estimé tout à fait opportun. Le BIT aurait donc également à composer avec la SGI, sans que fussent pour autant clairement établies les modalités de collaboration avec ce nouvel interlocuteur recommandé par le ministère ${ }^{19}$.

17. Voir, par exemple, son courrier du 24 octobre 1924 au représentant de l'Union des zemstvos de Belgrade qui se concluait ainsi « votre expérience nous sera alors des plus utiles en ce qui concerne le placement et le transfert des réfugiés» (ASDN, FMN, C 1437, Rr 404/3/22/1).

18. ASDN, FMN, C 1401, Rr 404/3/22/1, Lettre du 13 mars 1925 d'Albert Thomas au secrétaire du Comité directeur du Zemgor.

19. De fait, la SGI agit indépendamment du BIT et ne crut pas devoir informer Genève des recrutements effectués dans le secteur industriel. Les rapports des agents locaux du BIT permirent cependant de suivre, du moins partiellement, l'activité de la SGI (voir par exemple, ASDN, FMN, C 1401, R 402/10/55/1, rapport du 15 février 1926 du sous-délégué du BIT de Belgrade à ce sujet). 


\section{Le placement des Russes dans l'agriculture et l'élaboration du projet de colonisation agricole}

Au cours des pourparlers entre les services ministériels français et le BIT, Marcel Paon, chef du service de la main-d'œuvre du ministère de l'Agriculture, manifesta son intérêt pour le recrutement des réfugiés. Début 1925, il prit contact avec le BIT pour étudier la possibilité d'embaucher les réfugiés dans les exploitations agricoles françaises ${ }^{20}$. La pénurie de main-d'œuvre dans ce secteur était chronique et les Russes paraissaient particulièrement appropriés à la demande, comptant de nombreux cosaques, agriculteurs-soldats, sans parler des anciens exploitants et propriétaires terriens. Ces profils étaient fortement mis en valeur dans les rapports des organisations russes aux côtés des intellectuels estimés eux aussi très nombreux. Fort de cet intérêt, M. Paon se mit en relation avec les représentants du Zemgor pour évaluer les possibilités ${ }^{21}$. Au cours de ces entretiens, le fonctionnaire français fit part de son optimisme, évaluant de 70000 à 80000 le nombre de réfugiés qui pourraient être « absorbés » par l'agriculture 22 .

Il convient de souligner que les premières correspondances échangées entre l'administrateur français et le président du BIT au sujet du placement agricole des Russes se concentrèrent sur le rôle des organisations russes. A. Thomas fit un rappel à l'ordre en précisant les termes du modus vivendi établi dans la collaboration avec les Russes, auquel contrevenait ouvertement le responsable du ministère. Marcel Paon ne répondit pas sur le fond, mais distingua le Zemgor des autres organisations russes. Dans un courrier du 16 mars 1925, il fit savoir que les organisations militaires russes essayaient de faire recruter des contingents entiers.

Notre politique d'immigration, soulignait-il, me semblerait sérieusement battue en brèche par ces introductions clandestines de formations militaires sous le couvert de travaux agricoles ou industriels si elles devaient se continuer [...].

Pour en arriver à cette conclusion : « je pense que le Zemgor, qui lutte contre les tendances signalées, ne nous créera pas de surprises» ${ }^{23}$.

A. Thomas prit acte de la position de M. Paon, mais ne put s'empêcher de préciser :

J'ai une assez grande expérience des relations avec les Russes : nous ne serons jamais assez prudents. Sur tout il y a nécessité de montrer en toutes circonstances notre souci de coordination et de bon ordre. Ce sont des qualités qu'ils ignorent ${ }^{24}$.

20. ASDN, FMN, C 1411, R 405/2/22/1, Lettre de Marcel Paon au major Johnson du 16 mars 1925 se référant à une première rencontre de février 1925 à ce sujet.

21. Ibid., Télégramme d'A. Thomas du 17 mars 1925 signalant l'entrevue entre Marc Slonim, représentant du Zemgor de Prague, et M. Paon.

22. Ibid., Lettre de A. Thomas à M. Roques du 17 mars 1925 rapportant son entretien avec M. Slonim.

23. Ibid., Lettre de M. Paon à A. Thomas du 16 mars 1925.

24. Ibid., Lettre de A. Thomas à M. Paon du 19 mars 1925. 
La méfiance du président du BIT, même à l'égard du Zemgor dont il soutenait la collaboration, relevait donc d'une position de principe tout à fait explicite. Le parti pris de M. Paon en relevait également. Le chef du service de la main-d'œuvre à l'agriculture n'était pas un banal administrateur. Membre de la Ligue des droits de l'homme à une époque où celle-ci était très engagée en faveur de l'élaboration d'un statut juridique aux réfugiés, il abordait sa fonction avec la conviction du militant, prônant l'élaboration d'une politique d'immigration qui prît en compte l'intérêt d'État mais aussi celui des étrangers dans le respect de leur différence culturelle 25 . Cette posture prédisposait l'administrateur à un investissement spécifique dans la question des réfugiés et se traduisit en premier lieu par la volonté d'associer le Zemgor à l'entreprise de recrutement.

Ironie de l'histoire, c'est dans cette période de mise en œuvre du placement organisé que les craintes manifestées par M. Paon et relatives à l'immigration des militaires se concrétisèrent, non par le fait des organisations russes, mais par celui de la SGI ! L'agent du BIT à Belgrade signala que la SGI avait délivré par l'entremise exclusive du général Potocki, plus de 200 contrats aux réfugiés russes ${ }^{26}$. M. Paon réagit immédiatement :

Au cas où, malgré le présent avis, les dispositions prises par la SGI auraient pour but d'éliminer les groupements russes autres que celui du général Potocki, je vous serais obligé de m'en informer et à l'avenir je vous transmettrai directement s'il était nécessaire les contrats après les avoir visés au lieu de les remettre à la SGI27.

Tandis que le BIT voyait d'un mauvais œil la collaboration du Zemgor avec M. Paon, la SGI, de son côté, collaborait avec les organisations militaires au grand dam du responsable français. Malentendus et dysfonctionnement allaient s'accroître avec l'élaboration du nouveau projet de colonisation agricole.

Au terme de plusieurs mois d'efforts pour organiser le recrutement des réfugiés dans les exploitations françaises, M. Paon dressa un bilan très mitigé des opérations : les agriculteurs se montraient réticents à l'embauche de manœuvres russes. Le représentant français élabora alors un projet original et ambitieux : placer les Russes comme métayers et non comme ouvriers de ferme, les rendre maîtres de l'exploitation en favorisant ainsi leur reclassement ou leur promotion sociale ${ }^{28}$. Ce projet fut d'emblée présenté comme un vaste plan de colonisation

25. M. Paon livra sa position dans l'ouvrage qu'il publia l'année suivante et que préfaça A. Thomas (Marcel Paon, L'immigration en France, Paris : Payot, 1926).

26. Les responsables militaires constituaient effectivement des intermédiaires très pratiques pour les agents recruteurs. De plus, les vétérans de l'Armée blanche comptaient parmi eux de nombreux célibataires, fait important pour l'embauche dans l'industrie, les employeurs n'acceptant que de très faibles quotas de familles.

27. ASDN, FMN, C 1411, R 405/2/22/1, Lettre de Childs à M. Paon du 20 juin 1925 et réponse de ce dernier le 25 juin 1925 .

28. ASDN, FMN, C 1411, R 405/2/22/1, Correspondances de Roques (agent du BIT à Paris) à A. Thomas du 3 novembre 1925 et du 2 décembre 1925. 
agricole qui nécessitait d'importants ajustements : une réorganisation administrative des services départementaux de main-d'œuvre avec l'introduction de personnels russes ${ }^{29}$; l'établissement de contrats passés entre les propriétaires et le BIT, qui se portait caution du placement en métairies des réfugiés ; l'établissement, enfin, d'un système de prêts permettant d'assurer les frais de transport des futurs exploitants et leur subsistance pendant la période de «soudure » jusqu'à la première récolte ${ }^{30}$. Le projet rencontra l'adhésion enthousiaste et sans réserve du BIT comme du Zemgor. Depuis le début de la décennie, la colonisation agricole avait été défendue à Genève comme la seule vraie perspective d'insertion durable des réfugiés sans avoir pour autant rencontré de résultats probants. Elle s'avérait désormais à portée de la main. Comme le faisait remarquer l'agent du BIT à Paris : « on pourrait offrir aux réfugiés des conditions aussi avantageuses qu'au Canada et en Argentine et cette solution aurait l'intérêt d'économiser les frais de transports $»^{31}$. De son côté $\mathrm{A}$. Thomas rappelait à ses agents :

je demande au service des réfugiés russes d'examiner avec une très grande attention les propositions de M. Paon. Ce dernier est certainement mieux disposé que Lebelle à notre égard $32[\ldots]$. Il faut nous efforcer de garder toute son amitié et montrer qu'elle est bien placée ${ }^{33}$.

Dans ce projet, le BIT se trouvait au centre du dispositif, il ne jouait plus le simple rôle d'intermédiaire comme dans le cas du placement industriel. Les sollicitations dont il faisait l'objet confortaient le sens de sa mission et renforçaient sa position. Il en allait de même pour la mise en œuvre des prêts aux réfugiés dans laquelle il avait été demandé au BIT de s'impliquer ce, juste au moment où il s'apprêtait à solliciter à Genève l'instauration d'un fonds de roulement ${ }^{34}$. La demande s'en trouvait d'autant justifiée.

L'examen des aspects logistiques du projet s'étala sur la majeure partie de l'année 1926. L'entreprise de colonisation des réfugiés était conçue à titre d'essai dans le département du Gers ${ }^{35}$. Au cours de cette période, c'est le major Johnson qui devint au BIT le principal interlocuteur de Marcel Paon. Le major jouait un rôle clé à Genève en tant qu'homme de liaison entre le HCR et le BIT dont il avait, en

29. ASDN, FMN, C 1399, R 402/8/22/2, Compte-rendu de la rencontre avec M. Paon transmis par Roques (correspondant du BIT à Paris) à A. Thomas du 2 décembre 1925.

30. Ibid., Courrier de A. Thomas à M. Paon du 30 décembre 1925.

31. Ibid., Lettre de Roques à A. Thomas du 3 novembre 1925.

32. Ce dernier, en effet, collaborait activement avec la SGI au détriment du BIT.

33. ASDN, FMN, C 1411, R 405/2/22/1, Missive non datée (située dans des correspondances datant de la fin de l'année 1925).

34. Ibid., Courrier du major Johnson à Roques, avril 1926.

35. En choisissant ce département, il s'agissait moins d'un nouveau projet que de l'adaptation aux Russes d'un premier plan visant les Italiens. La désertification des régions du Sud-Ouest avait été à l'origine d'un important programme de placement en métairies des immigrés italiens qui n'avait toutefois pas rencontré le succès escompté en raison du manque d'intérêt témoigné à l'entreprise par les intéressés. 
quelque sorte, à valider l'action. Parmi les nombreux problèmes débattus avec le responsable du ministère de l'Agriculture, l'organisation des convois et celle du contrôle sanitaire des candidats firent l'objet de longues correspondances dans lesquelles le major Johnson fit valoir, de façon récurrente, l'intérêt qu'il y aurait à utiliser les services de la SGI. Cette puissante société avait établi plusieurs centres de sélection médicaux et sanitaires comme à Constantinople et elle recrutait beaucoup d'ouvriers en Pologne et en Yougoslavie auxquels on pouvait facilement adjoindre les convois de Russes recrutés dans ces pays ou dans les États baltes ${ }^{36}$. Contrairement au placement industriel où la recommandation de faire appel à la SGI provenait du responsable de la main-d'œuvre du ministère, dans le cas du placement agricole cette recommandation était faite par le principal interlocuteur au BIT. Sans être négatives, les réactions de M. Paon aux propositions de Genève restaient laconiques. Quant aux représentations russes locales, elles se plaignaient parfois explicitement de l'entremise de la SGI.

La question a été soulevée de savoir s'il n'était pas possible au BIT de (se passer) de l'intermédiaire des sociétés d'immigration ou de placement privées [...] qui travaillent presque toujours avec leurs propres agents et propres principes tout à fait commerciaux, principes qui ne sont pas ceux du BIT et ne prennent pas en compte les intérêts des réfugiés ${ }^{37}$.

En octobre 1926, dans un premier rapport peu concluant sur les offres des propriétaires du Gers, M. Paon proposait, de son côté, de renforcer l'implication des organisations russes dans le projet $^{38}$. Fallait-il y voir une façon d'annoncer les termes de la collaboration formelle engagée avec le Zemgor?

\section{L'instauration en France du partenariat avec le Zemgor}

Début novembre, Alexandre Konovaloff, président du Comité directeur du Zemgor à Paris informa A. Thomas de la création d'une «Commission pour l'établissement agricole des réfugiés russes », chargée de seconder le ministère dans ses efforts en matière de placement ${ }^{39}$.

36. ASDN, FMN C 1411, Rr 405/2/22/1, diverses correspondances du major Johnson à Roques et à M. Paon. Dans un courrier de mars 1925 à Roques, A. Thomas signalait déjà l'intérêt marqué du major Johnson pour une collaboration active avec la SGI « pour le placement surtout agricole des Russes » (C 1437, $\operatorname{Rr} 404 / 3 / 22 / 1)$.

37. ASDN, FMN C 1411, Rr 405/2/22/1, Rapport de Gallati (agent du BIT, correspondant de Pologne et des États baltes) à propos de la situation des réfugiés russes en Estonie, daté du $1^{\text {er }}$ novembre 1926.

38. Ibid., Lettre de M. Paon au major Johnson du 18 octobre 1926.

39. ASDN, FMN, C 1437, Rr 404/3/22/1, Lettre du 9 novembre 1926. Rappelons qu'au début des années 1920, il existait au Comité directeur un département de colonisation agricole qui gérait quelques exploitations prises en charge par les réfugiés dans le Bosphore. Voir Bjulleten' rossijskago zemsko-gorodskogo komiteta pomošči bežencam (année 1921). 
Quelle était cette commission? Son architecture était assez emblématique du mode de fonctionnement « des organisations russes ». Sous le label du Zemgor, elle coordonnait l'activité de différents réseaux formalisés à travers les associations. Le Zemgor de Prague (organisme agissant indépendamment du Zemgor de Paris) y était représenté et ce projet de colonisation agricole lui offrait surtout l'occasion de pouvoir placer les jeunes agronomes russes formés en Tchécoslovaquie ${ }^{40}$. Bien que prévenue de la saturation des offres d'emplois d'ingénieurs en France, l'organisation pragoise persévérait dans sa quête de débouchés pour les diplômés. L'Union générale des cosaques était également représentée à la Commission ; cet organisme fédérait une multitude d'associations localisées dans les différents pays de la diaspora et était ainsi à même de rassembler un nombre important de candidats au recrutement. L'alliance passée avec l'organisation cosaque concrétisait l'un des premiers objectifs du Zemgor qui était de favoriser la réinsertion des militaires à la vie civile. De ce point de vue, la colonisation agricole apparaissait comme la meilleure solution pour reclasser durablement les cosaques, nombreux dans l'émigration. La Commission comprenait encore la filiale « française » du Zemgor (Union des membres des zemstvos et des municipalités) qui, à travers l'animation des bureaux de travail, avait acquis une forte expérience en matière de recrutement. La nomination de Nicolas Avksentieff à la présidence de la Commission agricole témoigna de l'importance accordée par le Comité directeur parisien à la création de cette commission. Intellectuel réputé, membre du Comité directeur du Zemgor depuis sa création, N. Avksentieff avait derrière lui un long passé militant dans les rangs du parti socialiste révolutionnaire qui l'avait conduit aux premières loges de la vie politique russe en 1917-1918 (ministre de l'Intérieur du Gouvernement Provisoire à partir de juillet 1917, président du directoire d'Oufa de septembre à novembre 1918 , etc. ${ }^{41}$. Si l'activité politique et intellectuelle de N. Avksentieff faisait de cet homme une des grandes personnalités publiques de l'exil, son rôle dans l'assistance aux réfugiés demeura mal connu malgré l'énergie dont il fit preuve dans ses diverses fonctions. Durant les quatre années de sa présidence, N. Avksentieff s'investit intensément dans sa tâche. Tout en répondant aux multiples sollicitations du ministère de l'Agriculture, il recevait quotidiennement des chômeurs, rédigeait avec son secrétaire, M. Kommersant, de longues lettres de recommandation pour appuyer les candidatures à de simples emplois d'ouvriers de ferme, épluchait méthodiquement les rubriques d'offres d'emplois des nombreux journaux de province, etc. ${ }^{42}$.

40. Rappelons que le programme d'accueil des réfugiés russes mis en place par le gouvernement Masaryk au début de la décennie encourageait, notamment, l'immigration des intellectuels et des étudiants. Parmi les organismes de formation supérieure russes créés à Prague figurait l'Institut d'agriculture qui avait accueilli entre 1921 et 1928, 585 étudiants. C. Andreyev, I. Savický, Russia abroad. Prague and the Russian Diaspora, 1918-1938, New Haven, Londres : Yale University Press, 2004,p. 94.

41. J. Baynac, Les socialistes révolutionnaires, Paris : R. Laffont, 1979, p. 342-434 ; A. I. Serkov, Russkoe masonstvo 1731-2000 : Enciklopedičeskij slovar', Moscou : ROSSPEN, 2001, p. 39-40.

42. Archives Russes de Leeds, Zemel’naja komissija pri zemsko-gorodskom komitete, C-7982 (d'après le classement initial des archives du Comité directeur). 
La Commission disposait d'un bureau à Paris et salariait à ses frais un ingénieur agronome russe à Toulouse qui s'occupait de superviser l'établissement des fermiers et métayers dans la région. Au cours de ses deux premiers mois d'activité, elle était parvenue à placer 25 familles (soit 102 personnes), dont une partie dans les départements du Loiret et de la Seine-et-Marne ${ }^{43}$. Sa collaboration à l'installation des réfugiés dans le Sud-Ouest était comprise en somme comme une invitation à profiter de toutes les possibilités d'emplois dans l'agriculture.

Le rôle du Zemgor auprès du ministère de l'Agriculture apparut beaucoup plus clairement au cours des premiers mois de l'année 1927. Et pour cause ! Un tournant radical venait de s'opérer avec la suspension de l'immigration décrétée par le gouvernement français. Alors que les grands convois des réfugiés d'Europe centrale et orientale se préparaient enfin au départ, la France, soumise à une récession économique brutale, fermait ses frontières aux étrangers. Les réfugiés ne faisaient pas exception à la règle. Fin février, $\mathrm{M}$. Paon annonça la nouvelle mesure au major Johnson et lui exposa les dispositions envisagées pour parer l'échec du projet. Il rappelait que de nombreux réfugiés russes installés en France étaient licenciés des usines et voulaient « retourner à la terre ». Si le recrutement des Russes d'Europe centrale et orientale était effectivement suspendu, celui des réfugiés déjà installés en France pouvait prendre son essor. L'administrateur soulignait d'ailleurs que cette tâche absorbait déjà toutes les énergies de son service, mais qu'il la menait à bien «d'accord avec la Commission pour le placement agricole des réfugiés ». Pour ajouter immédiatement :

vous pouvez être tout à fait tranquille [...]. Je n'accepte pas de mener les questions concernant les réfugiés avec des organisations russes se trouvant à l'étranger et je leur déclare toujours que c'est sous votre égide que les opérations doivent se dérouler ${ }^{44}$.

Ce rappel des compétences soulignait le libre-arbitre avec lequel M. Paon entendait mener les opérations sur le territoire français. Tandis que le ministère faisait le choix d'un partenariat avec le Zemgor pour les recrutements «locaux », le BIT, avec la suspension des transferts, perdait une bonne partie de ses prérogatives et de ses moyens de contrôle sur l'action de placement. Les crispations liées à cette nouvelle situation ne se firent pas attendre. Entendant bien rester maître du recrutement, le major Johnson pria N. Avksentieff de transmettre à son agent en France (Roques) la liste des candidats qu'il pourrait soumettre au $\mathrm{BIT}^{45}$. La réaction fut immédiate et elle vint de M. Paon en personne qui fit comprendre au major Johnson qu'on n'avait pas besoin de ses services.

43. ASDN, FMN, C 1411, Rr 405/2/22/1, Rapport des activités de la Commission au 1er janvier 1927.

44. Ibid., Lettre de M. Paon au major Johnson du 28 février 1927.

45. Ibid., Lettre du major Johnson à N. Avksentieff du 12 mars 1927. 
Le placement se fait [...] par l'intermédiaire de mes agents à Toulouse [...] et ils recherchent d'accord avec mon service et d'accord avec le comité de placement agricole des réfugiés russes à Paris, des groupes pouvant convenir aux exploitations ${ }^{46}$.

En ce qui concernait le contrôle, il précisa qu'il ne rencontrait « aucune difficulté pratique ».

Son rôle en matière de recrutement conforta la position du Zemgor qui fit plusieurs suggestions pour préciser cette position et développer le projet de colonisation. La Commission agricole proposa ainsi la mise à disposition de ses services administratifs (deux secrétaires) pour gérer les modalités de remboursement des emprunts contractés par les réfugiés au BIT ${ }^{47}$. Au cours de l'année 1927, la Commission commença effectivement à assurer le suivi administratif de ces dossiers ${ }^{48}$. Au vu du caractère « très positif » de la collaboration « franco-russe », le BIT avalisa peu à peu la présence du Zemgor comme partenaire. Il donna bientôt son feu vert à la mise en place de contrôleurs russes auxiliaires auprès des services départementaux de main-d'œuvre.

En septembre 1927, le Zemgor précisait avoir reçu 13000 demandes de réfugiés et avoir placé 464 personnes à la tête de métairies (mais ne fournissait pas le nombre de ceux placés comme ouvriers de ferme $)^{49}$. Ses efforts étaient relayés par les agents départementaux du ministère. Dans un journal du Sud-Ouest, Pierre Andrieu, responsable du service de la main-d'œuvre locale, vantait les qualités des Russes :

ils constituent une main-d'œuvre intelligente, souple et opiniâtre, également très stable car ils considèrent leur retour en Russie comme problématique ${ }^{50}$.

«L'engouement pour la terre » qui se manifestait parmi les réfugiés conduisit les partenaires de la colonisation à examiner les possibilités de leur accès à la propriété. Les prêts nécessaires à ce type d'opération excédant les moyens du BIT, Marcel Paon envisagea alors un accord-cadre entre le Crédit agricole et le BIT, ce dernier se portant garant des réfugiés. Dans la présentation faite du projet au major Johnson

46. Ibid., Lettre de M. Paon au major Johnson du 14 mars 1927.

47. Ibid., Rapport sur l'activité de la Commission pour l'établissement agricole des réfugiés russes, 12 janvier 1927.

48. Curieusement, cependant, on ne trouve pas trace des modalités de l'accord dans les correspondances centrales. Mais cette activité est attestée par les archives administratives de la Commission agricole qui comportent une multitude de bordereaux et d'attestations de ce type : « les soussignés [...] reconnaissent avoir reçu de la Commission pour l'établissement des réfugiés russes la somme de 700 francs, 4e acompte sur le prêt de 7500 francs consenti par le HCR auprès de la SDN pour la mise en valeur de la métairie qu'ils exploitent » (Archives russes de Leeds, Zemel'naja komissija..., 1927-1929 et 1929-1931).

49. Présentation des activités de la Commission agricole au Conseil consultatif des organisations privées, Genève, 7 septembre 1927, ASDN, FMN C 1470, A/C-4-27.

50. ASDN, FMN C 1411 Rr 405/2/22/1, cité dans le rapport d'activité fait par la Commission agricole au BIT, le 2 décembre 1927. 
fin 1927, il ne cachait pas son optimisme: «je ne parle pas des possibilités d'absorption en France, elles sont presque illimitées $»^{51}$.

À la fin de l'année 1927, les tensions paraissaient dominées et les règles de la collaboration acceptées de tous. Pour parfaire l'embellie, la reprise de l'immigration se profilait, restaurant le rôle central du BIT dans les recrutements à distance. M. Paon mit cependant comme condition à cette reprise d'activité l'assurance d'une bonne sélection professionnelle effectuée sous l'égide des bureaux locaux du $\mathrm{BIT}^{52}$. La suite allait le montrer, le responsable du ministère visait là une collaboration active sur le terrain avec les représentants et les organisations russes de façon à éviter le recours à des services extérieurs ${ }^{53}$. Le message avait été entendu. Alors que E. Gallati, agent du BIT à Varsovie, rendait compte d'une proposition de collaboration de la SGI pour l'organisation des transports, le major Johnson lui répondit clairement « on peut utiliser le service de Duhamel (représentant de la SGI) si nous obtenons des contrats par son intermédiaire, mais si les contrats viennent d'autres sources (sous-entendu du ministère de l'Agriculture), son concours n'est pas souhaitable ${ }^{54}$. L'entremise du Zemgor, par le biais de ses filiales devint en revanche explicite dans les bureaux locaux. M. Serafimoff (sous-délégué en Bulgarie) rappela ainsi que le « recrutement à Sofia est assisté par le correspondant ici de la Commission agricole de l'Union des zemstvos et des villes russes, M. J. Zenkoff, qui est en même temps président du comité d'aide aux cosaques et possède en cette qualité plus de 100 représentants dans le pays $\gg^{55}$. Il faut reconnaître qu'à cette époque-là, dans la plupart des pays d'Europe centrale et orientale, les organisations russes étaient devenues des collaborateurs à part entière du recrutement. L'embauche se pratiquait sur la base d'une double médiation, d'une part le BIT et le ministère de l'Agriculture pour la gestion globale du système; d'autre part les associations russes pour l'organisation des transferts, tant sur les lieux de départs (sélection, contrôle) que sur ceux d'arrivées où la Commission agricole du Zemgor avait ses agents dans les services départementaux de main-d'œuvre. Les réfugiés concernés s'en remettaient à leurs autorités « naturelles ». La présence de celles-ci atténuait la relative dureté des conditions de « triage » (selon l'expression en vigueur), établissait un climat de confiance renforcé par la garantie d'un accueil à l'arrivée. Les réfugiés jouissaient ainsi, dans cette migration dirigée, d'un environnement humain privilégié que pouvaient leur envier bien des travailleurs polonais, yougoslaves ou italiens.

51. Ibid., Lettre de M. Paon au major Johnson du 23 novembre 1927.

52. Ibid.

53. Dans sa réponse au major Johnson le sous-délégué du BIT à Sofia, M. Serafimoff, soumettait ainsi son projet de création d'une commission de triage qui, outre un représentant du ministère bulgare de l'Agriculture, comportait un médecin de la Croix-Rouge russe, le responsable de l'Association russe des anciens combattants, le Comité d'aide aux cosaques, etc. Ibid., Lettre de M. Serafimoff au major Johnson du 30 décembre 1927.

54. Ibid., Lettre du major Johnson à Gallati du 22 février 1928.

55. ASDN, FMN, C 1455, Rr 412/110/22/3/1, Rapport de M. Serafimoff au service des réfugiés à Genève du 3 août 1928. 
Quels en étaient les bénéficiaires ? Les rapports effectués par les sous-délégués à Belgrade, Sofia, Varsovie mettent en évidence des profils très contrastés selon les pays. En Yougoslavie, les candidats étaient pour la plupart des cosaques peu instruits. Sur les 1200 cosaques du Don enregistrés, 700 n'avaient qu'une éducation primaire, 250 avaient poursuivi des études secondaires (sans avoir forcément l'équivalent du baccalauréat). 4000 des 5160 cosaques du Kouban, n'avaient qu'une éducation primaire, mais presque tous travaillaient dans l'agriculture en Serbie $^{56}$. En Bulgarie au contraire, le sous-délégué fournit des candidatures de réfugiés de formation supérieure, par exemple celle d'un certain M. Ivanoff « lui-même agronome de profession et de pratique, qui a été au service de l'État bulgare pendant plusieurs années, dirigeant une ferme d'état du sud de la Bulgarie ${ }^{57}$. Certains correspondants locaux insistaient sur l'important potentiel de maind'œuvre, d'autres sur la qualification des candidats et l'incidence de ces choix se ressentit dans les résultats. De fait, c'est avec Sofia que les transferts vers le SudOuest français furent les plus importants et les Russes de Bulgarie furent bientôt considérés comme les meilleures recrues ${ }^{58}$. Ils devaient surtout cette réputation à la stratégie adoptée par l'agent de Sofia, M. Serafimoff. Les très faibles recrutements effectués en Pologne pouvaient de la même manière être imputés au responsable local qui fit très tôt valoir que les réfugiés dans ce pays étaient particulièrement démunis et inorganisés, décourageant d'avance toute initiative d'envergure ${ }^{59}$. Une géographie préférentielle des recrutements fut ainsi clairement visible lors de la reprise de l'immigration. À en croire les appréciations portées par le chef du dépôt de Marseille qui relayait les opinions des interlocuteurs français, les candidatures venant de Constantinople ou de Pologne étaient jugées très peu fiables, contrairement à celles de Bulgarie et, dans une moindre mesure celles de Yougoslavie, qui étaient accueillies très favorablement.

La reprise de l'immigration fut également à l'origine d'un phénomène nouveau et prometteur. Les métayers russes installés en France commençaient à solliciter des contrats de travail à la ferme pour leurs compatriotes restés en Europe centrale et balkanique ${ }^{60}$. Un recrutement «interne » s'esquissait. Jamais les conditions de réalisation du projet n'avaient semblé aussi optimales. Pourtant, sous l'apparence d'un enthousiasme partagé, les relations entre le ministère et le BIT, ou plus préci-

56. ASDN, FMN, C 1447, $\operatorname{Rr}$ 410/86/2, Rapport (non daté) des demandes d'emplois de réfugiés russes dans l'agriculture en France.

57. ASDN, FMN, C 1455, Rr 412/110/23/3, Lettre de M. Serafimoff à Roques, du 22 février 1928.

58. La réputation acquise par la main-d'œuvre russe de Bulgarie est surtout perceptible dans les correspondances adressées au BIT par le chef du dépôt de Marseille (ASDN, FMN, C 1455, $\operatorname{Rr} 412 / 110 / 22 / 3)$. Il y eut cependant des plaintes concernant certains réfugiés sélectionnés jugés trop âgés ou incompétents.

59. ASDN, FMN, C 1411, R 405/2/22/1, Rapports de Gallati du 11 juin 1926, du 13 juillet 1926, etc. De fait, les recrutements de Pologne furent très faibles en général et en particulier lors de la reprise de l'immigration fin 1927.

60. ASDN, FMN, C 1455, Rr 412/110/22/3/1, Réponses de M. Serafimoff aux demandes de contrats (juin 1928-août 1929). 
sément entre M. Paon et le major Johnson, commençaient à singulièrement se détériorer. L'examen d'un projet de ferme modèle, qui allait exiger beaucoup d'énergie des différentes parties, révéla l'ampleur du différend.

\section{Apogée et abandon de la colonisation agricole}

Dans son programme de janvier 1927, la Commission agricole du Zemgor avait proposé la création d'une grande exploitation dans le Sud-Ouest, conçue comme un centre d'apprentissage : un grand nombre de réfugiés pourraient y séjourner avant de prendre une ferme en métayage. Le projet était ambitieux. Certes, il visait à assurer une formation technique (qui nécessitait l'emploi de plusieurs ingénieurs agronomes russes), mais il englobait également une formation linguistique et une initiation aux pratiques administratives françaises. Cette idée fit rapidement son chemin auprès des partenaires. Le major Johnson manifesta d'autant plus d'intérêt que le BIT se trouvait alors écarté des opérations de placement. Après un accueil positif, M. Paon, très absorbé par ses fonctions, marqua un certain découragement, mais il réactiva le projet avec l'essor de la colonisation début 1928. Dès lors, les échanges entre Genève et Paris se multiplièrent pour trouver le lieu adéquat dans le Sud-Ouest et concrétiser ainsi l'affaire. La première proposition avancée par le major Johnson sema d'emblée un certain désarroi. Celui-ci avait contacté la SGI qui avait mis sur pied une ferme modèle pour des métayers polonais. Se prévalant de ce précédent, le responsable au BIT soumit à M. Paon et au Zemgor une proposition de la SGI pour la création d'un centre de réadaptation agricole ${ }^{61}$. Dans sa réponse, N. Avksentieff souligna « qu'une œuvre de ce genre ne peut pas être organisée par une société commerciale privée comme la SGI », il précisait que seul le HCR, l'État français et les organisations russes « étaient susceptibles d'inspirer la confiance de l'opinion publique russe ${ }^{62}$. La réponse de M. Paon fut encore plus radicale. Après avoir rappelé que le projet nécessitait l'assentiment obligatoire du ministère, il poursuivait :

il est évident, que si la SGI doit recevoir une avance de 1250000 francs pour le capital d'exploitation et que si, d'autre part, elle absorbe pour frais de gestion tous les bénéfices de l'exploitation et l'intérêt du capital engagé en s'abstenant par ailleurs de payer les salaires aux ouvriers séjournant dans le centre, elle réalisera une bonne opération, mais le Haut commissariat en réalisera une mauvaise ${ }^{63}$.

Cela ne dissuada pas le major Johnson. Un mois plus tard, il confiait son enthousiasme au responsable de la SGI qui était en pourparlers avec le propriétaire du

61. ASDN, FMN, C 1453 Rr 412/5/22/2, Lettre du major Johnson à M. Paon du 16 mai 1928. En fin de courrier, l'auteur précisait qu'il avait également communiqué la proposition pour examen à la Commission agricole du Zemgor à Paris.

62. Ibid., Lettre de N. Avksentieff au major Johnson du 25 mai 1928.

63. Ibid., Lettre de M. Paon au major Johnson du 22 mai 1928. 
domaine de Bourepaux pour l'installation du Centre ${ }^{64}$. Une fois de plus, M. Paon réagit en soulignant que « des précautions spéciales seraient nécessaires si vous acceptiez de confier la gestion d'un centre à la SGI » ${ }^{65}$. L'implication des différents partenaires dans la recherche de l'exploitation prenait une tournure singulière. Tandis que le responsable du HCR-BIT ne jurait que par l'entremise de la SGI, Marcel Paon, lui, consacrait les premiers jours de ses « vacances à un voyage dans le Sud-Ouest en vue de rechercher l'exploitation agricole destinée à recevoir le centre d'adaptation ${ }^{66}$. À croire que le projet devenait une affaire personnelle ! Dès la fin du mois, le responsable du ministère soumettait une proposition au major Johnson. Le facteur temps, comme toujours dans les tractations relatives aux métairies, était l'une des contraintes majeures (il avait déjà contribué à faire échouer plusieurs projets de placements). Le contrat devait être impérativement signé avant la fin de l'automne de façon à pouvoir assurer l'ensemble du cycle de culture. Or le major Johnson répondit en précisant que tout en étant très intéressé par le projet, il ne serait pas disponible avant la fin octobre pour visiter les lieux et donner son accord éventuel ${ }^{67}$. Quelques jours plus tard, sans préciser la nature des obstacles, il faisait savoir à la SGI que sa proposition n'était pas retenue ${ }^{68}$. Ce scénario allait se renouveler jusqu'à la fin 1929. Les projets présentés par la SGI via le major Johnson étaient dénigrés par M. Paon. Pour ceux que présentaient M. Paon, le major Johnson bloquait les tractations par son indisponibilité chronique... Tandis que le projet de centre de rééducation agricole vacillait dans ce jeu stérile, la Commission agricole se concentrait sur la préparation du premier congrès de métayers russes. Dans l'esprit du Zemgor, la mobilisation des acteurs locaux et la médiatisation de l'événement devaient «permettre de lier les agriculteurs entre eux » et donner une nouvelle impulsion à la colonisation agricole ${ }^{69}$. Ce congrès tenu à la fin du mois de février 1929 à Toulouse rassembla quelques centaines de chefs d'exploitations sur les quelque 1500 installés dans la région dont, selon l'envoyé spécial des Poslednie novosti (Les Dernières nouvelles), les « cosaques du Don de la Garonne », les «cosaques du Kouban de la Dordogne », les «Voronejiens des Pyrénées $»^{70} \ldots$ Coquetterie de style du journaliste ou constat effectif de regroupements de gens de même pays au sein des différents départements ? Marcel Paon participa au congrès aux côtés des responsables régionaux des services de main-d'œuvre, de la Chambre d'agriculture, du Crédit agricole qui annonça à cette occasion l'accord tout juste signé avec le HCR pour l'attri-

64. «I am absolutly in agreement with the offer », soulignait-il d'emblée. Ibid., Lettre du major Johnson à Bonnet du 4 juin 1928

65. Ibid., Lettre de M. Paon au major Johnson du 19 juin 1928.

66. Ibid., Lettre de M. Paon au major Johnson du 25 juillet 1928.

67. Ibid., Lettre du major Johnson à M. Paon du 4 août 1928.

68. Ibid., Lettre du major Johnson à Bonnet du 9 août 1928.

69. Poslednie novosti, 6 février 1929.

70. Poslednie novosti, 20 février 1929. 
bution de prêts aux exploitants ${ }^{71}$. Le major Johnson se fit excuser. Au terme de ces trois journées de rencontre, Nicolas Avksentieff conclut sur une note optimiste annonçant, notamment, l'arrivée prochaine de réfugiés russes d'Estonie et la création d'un bulletin de liaison (édité par la Commission agricole) $)^{72}$. Le congrès, voulu comme une première grande étape dans l'essor de la colonisation agricole, marqua en réalité le point d'orgue de l'activité déployée en faveur des réfugiés. Dans le courant de l'année 1929, de nouvelles directives limitèrent l'immigration sur contrats avant de la suspendre complètement l'année suivante. Le bulletin de liaison ne connut que deux numéros et la Commission agricole du Zemgor cessa d'exister à l'aube de la nouvelle décennie ${ }^{73}$.

Comme l'ensemble des initiatives prises à l'égard des réfugiés russes, le projet de colonisation agricole appartient à l'histoire des années 1920, à cette décennie du «nouveau concert des Nations », portée par l'élan humaniste de l'après « der des ders » mais circonscrite par le brutal revers de la crise. La rétrospective du placement des réfugiés dans l'agriculture offre plusieurs grilles de lectures. Au vu du bilan, elle raconte l'histoire d'une entreprise chaotique, fragilisée par les aléas d'une conjoncture économique capricieuse, caractérisée par une extrême personnification des rôles, une démesure évidente entre l'énergie déployée et les résultats obtenus qui souligne, in fine, le bricolage désigné sous le terme d'action internationale. Quel bilan net peuton dresser de ces cinq années d'activité ? Il en ressort le placement dans l'agriculture de près de 3000 Russes, dont à peine la moitié comme exploitants, les autres comme ouvriers de ferme ; le recrutement d'une poignée de diplômés russes engagés comme auxiliaires dans les départements ; enfin, l'établissement d'un système de crédit. Les résultats, sans être négligeables, étaient bien en deçà de ceux escomptés.

Par ses divers paramètres, cette rétrospective se fait l'écho d'une micro-histoire qui fournit plusieurs éclairages, tant sur les protagonistes que sur la nature des conflits et des modes de fonctionnement de la coopération humanitaire. Celle-ci fut préfigurée par le Zemgor à travers son rôle pionnier dans le système d'embauche des réfugiés et l'utilisation qu'il fit de son réseau disséminé dans les pays d'accueil. Il faut souligner ici la capacité d'adaptation de l'organisation russe aux nouvelles formes de migrations et son pragmatisme dans l'action. Ceci peut être largement imputé aux parcours des animateurs sociaux de l'exil, anciens politiciens et agents de l'État russe qui ont conçu leur mission dans la continuité d'un service public au profit de la communauté exilée. La contribution des réfugiés russes à l'organisation de leur assistance internationale constitue ainsi un phénomène remarquable.

La confrontation entre les représentants institutionnels du projet sur le rôle des organisations et sociétés privées est spécifique à l'histoire de la colonisation agri-

71. Les prêts pouvaient aller jusqu'à un million de francs, mais les demandes devaient être soumises à l'accord préalable du HCR.

72. Les actes du congrès furent publiés par le Zemgor (Rossijsko-zemsko-gorodskoj komitet pomošči rossijskim graždanam zagranicej, Komissija po trudovomu zemel'nomu ustrojstvu russkih bežencev : otčet po soveščaniju russkih arendatorov na jugo-zapade Francii 16-18 fev. 1929 g. v Tuluze, sd, 75 p.).

73. Bjulleten' komissii po trudovomu zemel' nomu ustrojstvu bežentsev, juillet et août 1929. 
cole. On a pu observer tout au long de l'histoire du placement un glissement des rôles et des positions, induit par l'absence de règles clairement établies et la mise en œuvre de pratiques qui, sous l'apparence de l'improvisation, créèrent des situations de non-retour. Il en va ainsi de l'entremise de la SGI recommandée par le représentant du ministère du Travail, fait qui, pour cette raison, ne pouvait être ouvertement dénoncé par son homologue à l'Agriculture quand bien même le recours à cette Société fut par la suite défendu par le représentant du HCR-BIT. De son côté, M. Paon transgressa dès le début les principes adoptés relatifs au rôle attribué aux organisations russes. Les associations passées avec le secteur privé, bien qu'ayant été de nature très différente à Paris et Genève, ont toutes deux été revendiquées au nom de l'efficacité dans l'action. M. Paon faisait valoir le bénéfice des réseaux russes, le major Johnson, le professionnalisme de la SGI. C'est sur le terrain purement technique et logistique du placement que se sont déployés les rapports de force issus de ces alliances divergentes. Les considérations techniques et pragmatiques ont ainsi constitué les vecteurs privilégiés de la politisation du projet, révélant des conceptions opposées de l'humanitaire : d'une part, la normalisation de la situation des réfugiés par leur assimilation aux pratiques en vigueur dans l'immigration, de l'autre, l'adaptation des formules existantes à la situation spécifique des réfugiés. L'étroite imbrication des intérêts pragmatiques et politiques défendus par chacun des partenaires peut être observée à maintes reprises au cours des quatre années de coopération et elle s'est manifestée très explicitement au terme de la décennie, lorsque M. Paon fut nommé représentant du HCR en France ${ }^{74}$. Cette promotion ne découla pas tant de l'investissement personnel de l'administrateur en faveur des réfugiés que de la nécessité d'assurer le suivi du système d'emprunt mis en place avec le Crédit Agricole. Mais au-delà de cette fonction technique qui rendait incontestable la nomination de M. Paon, c'est la conception de l'action humanitaire du fonctionnaire français qui se trouvait avalisée. Les archives ne permettent pas de préciser la nature exacte de l'intérêt porté par le major Johnson à la coopération avec la SGI, mais les faits ultérieurs la suggèrent. Tandis que par la suite le major allait être démis de ses fonctions, la promotion de Marcel Paon comme homme de liaison entre Genève et les réfugiés en France signait le dénouement de la confrontation. Faute de résultats à la hauteur des attentes, les réfugiés russes avaient au moins gagné de leur investissement un allié de poids dans l'assistance.

Centre d'études des mondes russe, caucasien et centre-européen EHESS

Catherine.Gousseff@ehess.fr

74. Cette nomination intervint suite à l'arrangement international de 1928 qui sanctionna l'institutionnalisation des offices nationaux de réfugiés en les plaçant sous la tutelle du HCR (puis de l'Office Nansen qui lui succéda en 1930). La France signa 1'Arrangement, mais exigea que cette tutelle soit exercée par un fonctionnaire français, nommé par le gouvernement et agréé par Genève. Sur l'institutionnalisation des Offices de réfugiés, voir Catherine Gousseff, L'exil russe (1920-1939). Une histoire européenne, des trajectoires françaises, à paraître, 2006. 